\title{
An Efficient Electrochemical Tanks-in-Series Model for Lithium-Sulfur Batteries
}

\section{Caitlin D. Parke}

Department of Chemical Engineering, University of Washington, Seattle, WA 98195, USA

parkec3@uw.edu

Akshay Subramaniam

Department of Chemical Engineering, University of Washington, Seattle, WA 98195, USA

$\underline{\text { akshays1@uw.edu }}$

\section{Suryanarayana Kolluri}

Walker Department of Mechanical Engineering \& Material Science Engineering, Texas

Materials Institute, The University of Texas at Austin, Austin, TX 78712, USA

suryanarayanakolluri@utexas.edu

\section{Daniel T. Schwartz**}

Department of Chemical Engineering, University of Washington, Seattle, WA 98195, USA

dts@uw.edu

\section{Venkat R. Subramanian*,**}

Walker Department of Mechanical Engineering \& Material Science Engineering, Texas

Materials Institute, The University of Texas at Austin, Austin, TX 78712, USA

** ECS Fellow

*Corresponding Author, Fax: +1-512-471-8727

Email: venkat.subramanian@utexas.edu 


\begin{abstract}
This article applies and efficiently implements the Tanks-in-Series methodology (J. Electrochem. Soc., 167, 013534 (2020)) to generate a computationally efficient electrochemical model for Lithium Sulfur batteries. The original Tank model approach for Lithium-ion batteries is modified to account for porosity changes with time. In addition, exponential scaling is introduced that enables efficient simulation of the model equations to address a wide range of time constants present for different reactions in Lithium-sulfur system. The tank model achieves acceptable voltage error even for relatively aggressive conditions of discharge. Predictions of internal electrochemical variables are examined, and electrochemical implications of the approximations discussed. This suggests significant potential for real-time applications such as optimal charging, cell-balancing and estimation, and represents a step forward in efforts to incorporate detailed electrochemical models in advanced Battery Management Systems for Lithium-Sulfur batteries.
\end{abstract}




\section{Introduction}

Lithium sulfur (LiS) batteries have a high theoretical energy density of $2500 \mathrm{Wh} / \mathrm{kg}$, and in recent years, the research on these batteries has increased dramatically ${ }^{1}$. Despite their promise, lithium sulfur batteries suffer from several challenges, ranging from the cycling stability of lithium metal to the insulating nature of the sulfur cathode which limits power capabilities.

Mathematical modeling can help accelerate commercialization of LiS batteries by elucidating the underlying mechanisms and enabling the exploration of the design space without the time and cost of experiments. Electrochemical physics-based models offer insight into the internal states of the battery, such as local overpotentials and concentrations throughout a cell. The first physics-based model for lithium sulfur batteries was a zero-dimensional lumped model published in 2004 by Mikhaylik and Akridge ${ }^{2}$, which included simple kinetics and thermodynamics. In 2008, Kumaresan et al. ${ }^{3}$ published a one-dimensional (1D) mathematical model for the discharge of LiSbatteries. This model included transport effects, a more realistic set of sulfur reduction chemistries, and morphological changes in the cathode. Diffusion effects associated with soluble species and precipitation of insoluble species in the cathode were captured through a changing porosity. A related model was developed by Neidhardt et al. ${ }^{4}$ in 2012. A series of papers were published with a similar 1D model that explored impedance ${ }^{5}$, charging $^{6}$, and degradation ${ }^{7-9}$, as well as lumped models with simplified reaction mechanisms, reduced number of parameters ${ }^{10-12}$, and precipitation of lithium sulfide ${ }^{13,14}$.

For all models, there is an inherent tradeoff with computational efficiency, ease of parameterization, and model complexity. Within the literature on lithium sulfur models, the 1D models are more descriptive and can capture experimentally-observed voltage characteristics 
reasonably well, such as the relative length and potential of the typical two-plateau discharge curve and the "nucleation overpotential" between the two plateaus upon precipitate formation. Simplified models have been developed specifically for increased computational efficiency and ease of parameterization, and they have been used to predict general trends of the second voltage plateau rather than the overall shape of voltage curves ${ }^{11,12,15,16}$. Although simpler models have faster solution times, the power of using physics-based models is the insight they provide into the internal states that contribute to cell performance and the direct linkage to the geometric, chemical, and physical properties of the electrodes, electrolyte, and separator.

To predict battery performance, it is vital that the parameters of the model are calibrated to experiments through estimation of parameters. Unfortunately, experimental validation of lithium sulfur models is still lacking in the literature as the chemistry is relatively new and collection of performance data at different operating protocols for different rates is very expensive and rare. Furthermore, system dynamics, including reaction mechanisms, are very sensitive to the electrolyte ${ }^{17}$, which highlights the importance of parameter estimation to improve individual system predictions. Leveraging the prediction power of the $1 \mathrm{D}$ models for parameter estimation can enable better agreement between models and experiments.

Another application of physics-based models is design space exploration and optimization. In general, the physics-based battery models are highly non-linear and stiff systems. Optimization of parameters can mean hundreds to thousands of model calls (in sequential optimization framework which is the most commonly used approach in the optimization community), which quickly can become computationally intractable ${ }^{18}$. Fast and robust models that maintain high predictive capabilities can accelerate research by allowing greater exploration of design space and optimization with efficiency. Both parameter estimation 
and exploration of design space underpin the importance of efficient yet descriptive models.

This work focuses on developing an efficient physics-based model that maintains similar predictive capabilities to $1 \mathrm{D}$ models under certain conditions while also ensuring computational efficiency for parameter estimation, real-time control, and design. The tank-in-series model presented here is based on the $1 \mathrm{D} \mathrm{Kumaresan}$ model $^{3}$ and does not include extra mechanisms, thereby allowing this paper to focus on further development of the volume-averaging method. This framework is flexible, and more physics and chemistry can be added as needed while maintaining model efficiency. 


\section{Mathematical and Experimental Methods}

The following reactions describe the discharge of a lithium sulfur battery.

$$
\begin{gathered}
L i_{(s)} \rightleftharpoons L i^{+}+e^{-} \\
\frac{1}{2} S_{8(l)}+e^{-} \rightleftharpoons \frac{1}{2} S_{8}^{2-} \\
\frac{3}{2} S_{8}^{2-}+e^{-} \rightleftharpoons 2 S_{6}^{2-} \\
S_{6}^{2-}+e^{-} \rightleftharpoons \frac{3}{2} S_{4}^{2-} \\
\frac{1}{2} S_{4}^{2-}+e^{-} \rightleftharpoons S_{2}^{2-} \\
\frac{1}{2} S_{2}^{2-}+e^{-} \rightleftharpoons S^{2-} \\
S_{8(s)} \rightleftharpoons S_{8(l)} \\
2 L i^{+}+S^{2-} \rightleftharpoons 2 L i_{2} S_{(s)}
\end{gathered}
$$

Reaction (1) describes the oxidation of the lithium foil anode. Reactions (2)-(6) refer to the electrochemical reduction of liquid S8 in the cathode. Reactions (7) and (8) are the dissolution and precipitation of the two solid species, $\mathrm{S}_{8(\mathrm{~s})}$ and $\mathrm{Li}_{2} \mathrm{~S}_{(\mathrm{s})}$. The work assumes the same reaction mechanism as the Kumaresan model $^{3}$. However, $\mathrm{Li}_{2} \mathrm{~S}$ is the only solid product considered as the presence of other precipitates is still debated ${ }^{9,10,19}$.

Figure 1 (a) is the physical representation of lithium sulfur batteries, which consists of the composite cathode made of yellow solid sulfur and black conductive carbon, the porous 
separator in blue, and the anode made of lithium foil in grey. Because the focus of this work is primarily the sulfur cathode dynamics, the lithium anode has been included as a constant source of lithium ions without furthering considering side reactions or limitations.

The governing equations, shown in Table I, are taken from the one-dimensional (1D) mathematical model by Kumaresan et $\mathrm{al}^{3}$. The model assumes dilute solution theory that does not consider the effect of species' interactions on diffusion. Each part of the voltage curve can be attributed to the physical phenomena that is happening during discharge, which physics-based models capture. The Kumaresan and White model includes transport by migration and diffusion, Butler-Volmer kinetics, thermodynamics described by Nernst equations, and morphology changes in the cathode. The porosity and volume fractions of solid sulfur and lithium sulfide change with time, and the available volume of the cathode then directly affects the diffusion of the ionic species. Both electrochemical and chemical reactions are present in the model as there are both reduction reactions involving sulfur species and non-charge transfer dissolution and precipitation reactions. The parameters used in this work are included in Tables Table II-Table $\mathrm{V}$, which have been modified from the base parameters in the Kumaresan model. Where indicated, the parameters reflect the best fit from parameter estimation to experimental data from a C/5 discharge curve. The effect of the diffusion constants, the cathode thickness, and the exchange current densities in particular is considered in the Discussion, and the parameter values listed here represent the baseline. 


\section{Model Development}

In this section, we propose and discuss the development of the mass-conserving volumeaveraged tank-in-series model for lithium sulfur batteries, included in Table VI. In the tank model, each region is approximated as a tank connected in series. Previously, our group has developed and validated this approach for Lithium-ion batteries ${ }^{20}$, and one of the key differences and challenges is the dynamic porosity within lithium sulfur batteries. The equations are spatially integrated over the two regions, the cathode and the separator. The anode is modeled as a constant flux boundary condition for lithium ions. The result is average variables in each region, and the midpoint approximation is used to calculate interfacial fluxes and species between the cathode and separator. The cathode and separator are treated as separate tanks as depicted in Figure 3, and the equations for each variable is volume averaged over that region. Through the use of the flux boundary conditions, the mass flow between the tanks can be coupled.

To illustrate volume averaging, the sulfide ion, $S^{2-}$, the final ionic species in the reduction scheme is considered. The governing equations for the cathode, Equation (9), is written for $S^{2-}$. In the cathode, the governing equation for the sulfide ions is

$$
\frac{\partial \varepsilon_{1} C_{1, S}}{\partial t}=-\frac{\partial N_{1, S}}{\partial x}+r_{S}-R_{1, S} \quad 0<x<l_{1}
$$

where $\varepsilon_{1}$ denotes the porosity in region 1 or the cathode, $C_{1, S}$ is the concentration of $S^{2-}$ in the cathode, $r_{s}$ is the rate of the production of all of the electrochemical reactions involving $S^{2-}$, $R_{1, S}$ is the rate of consumption of the precipitation reaction in the cathode for $S^{2-}$. For subscripts on variables, 1 denotes a variable in the cathode or region 1 , and 2 is the separator or region 2 . 
The subscript $S$ denotes the $\mathrm{S}^{2-}$ species. $N_{1, S}$ represents the flux in the cathode, which considers both diffusion and migration, defined as

$$
\frac{N_{1, S}}{\varepsilon_{1}}=-D_{S} \frac{\partial C_{1, S}}{\partial x}-z_{S} \frac{D_{S}}{R T} F C_{1, S} \frac{\partial \phi_{1, e}}{\partial x}
$$

where $D_{S}$ is the diffusion coefficient corrected by the Bruggeman expression where $D_{i}=D_{i, 0} \varepsilon^{b}$, $z_{S}$ is the charge number of $S^{2-}, \phi_{1, e}$ is the electrolyte-phase potential in the cathode.

Similarly, in the separator, the overall species balance is

$$
\frac{\partial \varepsilon_{2} C_{2, S}}{\partial t}=-\frac{\partial N_{2, S}}{\partial x}-R_{2, S} \quad l_{1}<x<l_{1}+l_{2}
$$

Where $N_{2, S}$ is defined as

$$
\frac{N_{2, S}}{\varepsilon_{2}}=-D_{S} \frac{\partial C_{2, S}}{\partial x}-z_{S} \frac{D_{S}}{R T} F C_{2, S} \frac{\partial \phi_{2, e}}{\partial x}
$$

The flux boundary conditions for sulfide ions are

$$
\begin{array}{lr}
N_{0 / 1, S}=0 & x=0(13) \\
N_{2 / 3, S}=0 & x=l_{1}+l_{2}(14)
\end{array}
$$

Where $0 / 1$ indicates the interface of the cathode and the current collector and $2 / 3$ indicates the interface between the separator and the lithium foil anode. Between the cathode and separator, continuity of fluxes is maintained.

The governing equations are then volume averaged by integrating over the volume of each region. For illustration, the governing equation for $S^{2-}$ is integrated over the volume, $V_{l}$, in Equation 15.

$$
\frac{d \int_{V_{1}} \varepsilon_{1} C_{1, S} d V}{d t}=-\int_{V_{1}} \frac{\partial N_{1, S}}{\partial x} d V+\int_{V_{1}} r_{S} d V-\int_{V_{1}} R_{1, S} d V
$$


In the one-dimensional model, the volume integral is over the differential volume $d V$, which is considered to be $d V=A d x$, where $A$ is a constant cross-sectional area. For each average quantity, the integral is expressed as $\overline{C_{1, i}}=\frac{\int_{V_{1}} C_{1, i} d V}{\int_{V_{1}} d V}, \overline{r_{i}}=\frac{\int_{V_{1}} r_{i} d V}{\int_{V_{1}} d V}$, and $\overline{R_{1, i}}=\frac{\int_{V_{1}} R_{1, i} d V}{\int_{V_{1}} d V}$, with $\bar{v}$ denoting the volume average of variable $v$ in the given region or 'tank'. Substituting these relations back into the governing equation gives a one-dimensional integral over the length of the cathode

$$
\frac{d\left(\overline{\varepsilon_{1}} \overline{C_{1, S}}\right)}{d t}=\frac{\int_{x=0}^{x=l_{1}} \frac{\partial N_{1, S}}{\partial x} d x}{l_{1}}+\overline{r_{S}}-\overline{R_{1, S}}=\frac{N_{1, S, x=0}-N_{1, S, x=l_{p}}}{l_{1}}+\overline{r_{S}}-\overline{R_{1, S}}
$$

Substituting $N_{1, S, x=0}=0$ into the previous equation, results in

$$
\frac{d\left(\overline{\varepsilon_{1}} \overline{C_{1, S}}\right)}{d t}=\frac{-N_{1, S, x=l_{1}}}{l_{1}}+\overline{r_{S}}-\overline{R_{1, S}}
$$

The same can be done for the separator to arrive at the following with the substitution $N_{2, S, x=l_{1}+l_{2}}=0$

$$
\frac{d\left(\overline{\varepsilon_{2}} \overline{C_{2, S}}\right)}{d t}=\frac{N_{2, S, x=l_{1}}}{l_{2}}-\overline{R_{2, S}}
$$

Next, let us consider how to approximate the flux at the interface of the cathode and the separator. Through the use of the flux boundary conditions, the mass flow between the tanks can be coupled. For the approximation, a 'driving force' $\Delta C_{m}$ and a 'length scale' $\delta_{m}$ is defined for interface between the separator and cathode, where subscript $m$ denotes 1 for cathode or 2 for separator. The 'driving force' $\Delta C_{m}$ is the difference between the average concentration of the region and the unknown concentration at the interface $\Delta C_{m}=\overline{C_{m}}-C_{x=\text { interface }}$. The 'length scale' 
is $\delta_{m}$, defined by $\delta_{m}=\delta \cdot l_{m}$, where $\delta$ is the dimensionless fraction where concentration gradients are assumed to be, and $l_{m}$ is the thickness of the domain. Note, the variable delta will be discussed throughout the Results and Discussion to consider the effect of the length scale approximation has on the tank model predictions.

Similarly, a 'driving force' $\Delta \phi_{e}$ can be defined for the liquid potential that is approximated as $\Delta \phi_{e}=\overline{\phi_{e}}-\phi_{e, x=\text { interface }}$. For each concentration, the interfacial boundary conditions from each region can be equated to determine the unknown value at the interface. For these approximations, the error scales with the square of the domain thickness. The resulting flux equation becomes

$$
\begin{aligned}
& N_{1, S, x=l_{1}}=-D_{S} \varepsilon_{1} \frac{\partial C_{1, S}}{\partial x}{ }_{x=l_{1}}-z_{S} \frac{D_{S}}{R T} \varepsilon_{1} F C_{1, S} \frac{\partial \phi_{1, e}}{\partial x} \cong-D_{S} \varepsilon_{1} \frac{\Delta C_{1}}{\delta_{1}}-z_{S} \frac{D_{S}}{R T} F \varepsilon_{1} C_{1, S} \frac{\Delta \phi_{1, e}}{\delta_{1}} \\
& =-D_{S} \overline{\varepsilon_{1}}\left(\frac{\overline{C_{1, S}}-C_{1, S, x=l_{1}}}{\delta_{1}}\right)-z_{S} \frac{D_{S}}{R T} F \overline{\varepsilon_{1}} \overline{C_{1, S}}\left(\frac{\overline{\phi_{1, e}}-\phi_{1, e x=l_{1}}}{\delta_{1}}\right)
\end{aligned}
$$

A similar expression can be written for the interfacial flux from the separator.

$$
\begin{aligned}
& N_{2, S, x=l_{1}}=-D_{S} \varepsilon_{2}{\frac{\partial C_{2, S}}{\partial x}}_{x=l_{1}}-z_{S} \frac{D_{S}}{R T} \varepsilon_{2} F C_{2, S} \frac{\partial \phi_{2, e}}{\partial x} \underset{x=l_{1}}{\cong}-D_{S} \varepsilon_{2} \frac{\Delta C_{2}}{\delta_{2}}-z_{S} \frac{D_{S}}{R T} F \varepsilon_{2} C_{2, S} \frac{\Delta \phi_{2, e}}{\delta_{2}} \\
& =-D_{S} \overline{\varepsilon_{2}}\left(\frac{-\overline{C_{2, S}}+C_{2, S, x=l_{1}}}{\delta_{2}}\right)-z_{S} \frac{D_{S}}{R T} F \overline{\varepsilon_{2}} \overline{C_{2, S}}\left(\frac{-\overline{\phi_{2, e}}+\phi_{2, e, x=l_{1}}}{\delta_{2}}\right)
\end{aligned}
$$

The electroneutrality expression is given by

$$
\sum_{i} z_{i} C_{i}=0
$$

Electroneutrality provides the equation for the potential. Using this expression to eliminate one of the concentration variables and substituting back into the boundary conditions at the interface $N_{1, i, x=l_{1}}=N_{2, i, x=l_{1}}$, the boundary conditions simplify to 


$$
\varepsilon_{1}{\frac{\partial \phi_{1, e}}{\partial x} \quad x=l_{1}}=\varepsilon_{2}{\frac{\partial \phi_{2, e}}{\partial x}}_{x=l_{1}}
$$

This can be approximated as

$$
\varepsilon_{1}{\frac{\Delta \phi_{1, e}}{\delta_{1}}}_{x=l_{1}}=\varepsilon_{2}{\frac{\Delta \phi_{2, e}}{\delta_{2}}}_{x=l_{1}}
$$

Which can be simplified as

$$
\overline{\varepsilon_{1}} \frac{\phi_{1, e, x=l_{1}}-\overline{\phi_{1, e}}}{\delta_{1}}=\overline{\varepsilon_{2}} \frac{\overline{\phi_{2, e}}-\phi_{2, e, x=l_{1}}}{\delta_{2}}
$$

Next, we can use the interfacial boundary condition of continuity where $\phi_{1, e, x=l_{1}}=\phi_{2, e, x=l_{1}}=\phi_{e, \text { mid }}$ to simplify Equation 24.

$$
\phi_{e, \text { mid }}=\frac{\overline{\varepsilon_{1}} \delta_{2} \overline{\phi_{1, e}}+\overline{\varepsilon_{2}} \delta_{1} \overline{\phi_{2, e}}}{\overline{\varepsilon_{1}} \delta_{2}+\overline{\varepsilon_{2}} \delta_{1}}
$$

Similarly, for concentration, simplification of the flux conditions at the interface results in

$$
\varepsilon_{1}{\frac{\partial C_{1, i}}{\partial x_{x=l_{1}}}}_{=\varepsilon_{2}}{\frac{\partial C_{2, i}}{\partial x_{x=l_{1}}}}
$$

Where after substituting the 'driving force' and 'diffusion length' expression becomes

$$
C_{m i d, S}=\frac{\delta_{2} \overline{\varepsilon_{1}} \overline{C_{1, S}}+\delta_{1} \overline{\varepsilon_{2}} \overline{C_{2, S}}}{\overline{\varepsilon_{1}} \delta_{2}+\overline{\varepsilon_{2}} \delta_{1}}
$$

Now that we have defined the interfacial quantities for concentration and liquid-phase potential, the flux at the interface can be determined.

$$
N_{1, S, x=l_{1}}=-D_{S} \overline{\varepsilon_{1}}\left(\frac{\overline{C_{1, S}}-C_{m i d, S}}{\delta_{1}}\right)-z_{S} \frac{D_{S}}{R T} F \overline{\varepsilon_{1}} \overline{C_{1, S}}\left(\frac{\overline{\phi_{1, e}}-\phi_{e, \text { mid }}}{\delta_{1}}\right)
$$




$$
N_{2, S, x=l_{1}}=-D_{S} \overline{\varepsilon_{2}}\left(\frac{-\overline{C_{2, S}}+C_{\text {mid }, S}}{\delta_{2}}\right)-z_{S} \frac{D_{S}}{R T} F \overline{\varepsilon_{2}} \overline{C_{2, S}}\left(\frac{-\overline{\phi_{2, e}}+\phi_{e, m i d}}{\delta_{2}}\right)
$$

Let us now consider the potential equations, and in this case, the current equations are used instead of explicit potential equations. Let us consider the equation for conservation of charge.

$$
\frac{\partial i_{s}}{\partial x}+\frac{\partial i_{e}}{\partial x}=0
$$

Where $i_{s}$ is the current within the solid phase and $i_{e}$ is current in the liquid phase.

After substituting in $\frac{\partial i_{e}}{\partial x}=a \sum_{j} i_{j}$ for the liquid phase current term, we arrive at the following

$$
\frac{\partial i_{s}}{\partial x}+a \sum_{j} i_{j}=0
$$

Through volume averaging and substituting the boundary conditions for the solid phase current, the following expression can be determined

$$
-\frac{i_{s, x=l_{1}}-i_{s, x=0}}{l_{1}}=-\frac{0-i_{a p p}}{l_{1}}=\frac{i_{a p p}}{l_{1}}=a \sum_{j} i_{j}
$$

For the liquid phase potential, we start with the electroneutrality expression, Equation 19, and multiply by the porosity, $\varepsilon$, and take the derivative with respect to time

$$
\sum_{i} z_{i} \frac{d\left(\bar{\varepsilon} \overline{C_{i}}\right)}{d t}=0
$$

Then, substitute the mass balance expression for the term $\frac{d\left(\bar{\varepsilon} \overline{C_{i}}\right)}{d t}$ for the liquid phase potential equation. This results in the system of nonlinear differential algebraic equations (DAE) shown in Table VI.

Typically DAE models are written in the form $d y / d t=f(y, g), O=g(y, g)$, where $y$ is the 
variable mapped to the differential equations and $g$ is the variable mapped to the algebraic equations. This system can be solved using any DAE solver in time such as IDA in C, DASKR in fortran, ode15s or ode15i in MATLAB, etc. However, because of varying dynamics in the system and the multiscale nature of different time constants and reactions, most adaptive solvers cannot simulate beyond 2.0V during discharge. For example, a past MATLAB code (simple scheme listed elsewhere ${ }^{15}$ ) fails when the solver reaches $1.9 \mathrm{~V}$. In this paper, we propose an effective scaling methodology to scale multiple variables. The equation for concentration is of the form is $d C / d t=a C+b$, and we define $\log$ scale as $\hat{C}=\ln (C)$. The scaled variables are species concentrations, volume fractions, and cathode porosity while the potentials are not scaled. With the combined time scaling and the averaging of the spatial variables, the tank model consistently reaches the voltage cutoff whereas a singularity occurs in the 1D model at the end of discharge for most conditions. This scaling helps the original 1D model as well.

To illustrate the time scaling, let us consider the $\mathrm{S}^{2-}$ species again. Considering the mass balance in the cathode, substituting $C=\ln (\bar{C})$ for each average variable results in

$$
\frac{d\left(\varepsilon_{1} C_{1, S}\right)}{d t}=\frac{-N_{1, S, x=l_{1}}}{l_{1}}+\overline{r_{S}}-\overline{R_{1, S}}
$$

Where the flux is now

$$
N_{1, S, x=l_{1}}=-D_{S} \varepsilon_{1}\left(\frac{C_{1, S}-C_{m i d, S}}{\delta_{1}}\right)-z_{S} \frac{D_{S}}{R T} F \varepsilon_{1} C_{1, S}\left(\frac{\overline{\phi_{1, e}}-\phi_{e, \text { mid }}}{\delta_{1}}\right)
$$

Notice that the potential variables have not been substituted because they are algebraic variables instead of differential variables, and their range is on the order of 1 and does not change over orders of magnitude. The substitution can be done for the rest of the equations to arrive at the set 
equations found in Table VII.

The resulting system of equations was written in MAPLE and solved with its stiff solver based on semi-implicit runge kutta solver called through the dsolve command in implicit mode. A robust initialization strategy was used to find consistent initial conditions ${ }^{21}$. The same set of equations were also solved in IDA which enables order of magnitude faster computation compared to Maple.

Experimental data is from the first discharge cycle of a proprietary, pre-commercial 19.5 Ah Li-S pouch cell. The C/5 discharge was performed using an Arbin high current battery cycler with cell temperature of $20^{\circ} \mathrm{C}$. Base-case parameters presented in Tables Table II-Table V were derived from this discharge data. 


\section{Results and Discussions}

The tank-in-series model (tank model) was developed through volume averaging of the one-dimensional model (1D model), which necessitated making assumptions. The dimensionless

parameter $\delta$, where $\delta=\delta_{l} / l_{l}$, i.e., the fraction of the cathode and the separator, was introduced to describe the approximate length scale where gradients occur within the domain. To understand how well the tank model performs in comparison with the 1D model, a preliminary parametric study was performed through varying the $\mathrm{C}$ rate, diffusion coefficients, the cathode thickness, and exchange current densities, listed in Table VIII. The three different rates considered are $0.2 \mathrm{C}, 0.5 \mathrm{C}$, and $1 \mathrm{C}$, which are appropriate for the energy demands for electric vehicles ${ }^{22}$. The diffusion coefficient for all ionic species except the anion was assumed, to simplify the model, while the anion was assumed to be a typical lithium salt, such as bis(trifluoromethane)sulfonimide (TFSI-), with an appropriate diffusion coefficient ${ }^{23}$. The cathode thickness included the base case from the Kumaresan model and twice the base case. The exchange current densities for each reaction were varied orders of magnitude above and below the base case; the results follow the general trends discussed below and are not shown.

Results with the base case parameters are shown in Figure 2 (a). For this parameter set and discharge rates, the 1D model shows there are negligible transport limitations in the Li-S cell. That means species concentrations and partial currents are fairly uniform across the electrode, reducing the underlying cause of distinctions between the tank model and the 1D model. In this limiting case of negligible transport, we expect the tank model to match the more complete 1D model, and this is exactly what we see in Figure 2 (a). Convergence between two 
independent models under appropriate limiting conditions is good evidence for computational accuracy.

The tank model discharge curve in Fig. 2a took 40 ms of CPU time on an Intel (R) Xeon (R) E-2176M processor with a clock speed $2.70 \mathrm{GHz}$ and $64 \mathrm{~GB}$ RAM, whereas the 1D model took at least 10x more time. The benefits of this computational speed up is critically felt in model-based parameter estimation or optimal design, where thousands of model call are implemented. To have confidence using this fast-computing tank model for parameter estimation or optimal design outside of the negligible transport-limits, we explore parameters and operating rates that induce concentration gradients within the cell, allowing us to probe the tank model suitability (compared to the 1D model) under more challenging conditions.

To explore the assumption of uniform concentration, the diffusion coefficient was varied over 3 orders of magnitude, which resulted in different transport effects on the solution. For the voltage curves in Figure 2 (a). , the diffusion coefficient of $1 \times 10^{-10} \mathrm{~m}^{2} / \mathrm{s}$ was used in the tank model and the 1D model, which match at all rates, indicating that the concentration profile is in fact uniform. To quantify the variation in concentration across the cathode, we can calculate a spread of the soluble sulfur equivalents and normalize by the average concentration, resulting in $42 \%\left(\Delta \mathrm{C} / \mathrm{C}_{\text {avg }} \times 100 \%\right)$ for the $1 \mathrm{D}$ model at the $1 \mathrm{C}$ rate at $95 \%$ of total discharge. The diffusion coefficients are the same order of magnitude as the base parameters from the Kumaresan paper. Work by Zhang et $\mathrm{al}^{9}$ show that the original Kumaresan model parameters are kinetically-limited and do not capture rate capability well at higher discharge rates. Their work found that changing the ionic diffusion coefficients by one to two orders of magnitude, the model was able to predict experimental data more accurately under varying rates.

Next, let us consider the next set of simulations with diffusion coefficient of $1 \times 10^{-11} \mathrm{~m}^{2} / \mathrm{s}$ 
to introduce additional transport limitations, see Figure 2 (b). . At $0.2 \mathrm{C}$ and $0.5 \mathrm{C}$ rates, the difference in predictions between the $1 \mathrm{D}$ and tank models is negligible. The simulations at $1 \mathrm{C}$ indicate the overall voltage response is closely matched until the end of discharge, where there is some slight deviation. Lowering the diffusion coefficient to $1 \times 10^{-11} \mathrm{~m}^{2} / \mathrm{s}$ increases transport limitations, as indirectly evidenced by the reduction in overall capacity as a function of rate. The more direct measure of increasing transport limitations is the variation in concentration across the cathode, which is $287 \%\left(\Delta \mathrm{C} / \mathrm{C}_{\mathrm{avg}} \times 100 \%\right)$ for the $1 \mathrm{C}$ rate in the $1 \mathrm{D}$ model for normalized soluble sulfur equivalents at $95 \%$ of discharge. In Figure 2 , a diffusion length of $1 / 3^{\text {rd }}$ the domain thickness is used to best match the 1D model predictions.

As we decrease the diffusion coefficient by an additional order of magnitude, further accentuating transport effects, the effect of the tank model parameter $\delta$ becomes more important (Figure 3). This extra parameter, $\delta$, is an approximate diffusion length that can be seen as a transport fitting parameter. Under conditions where transport limitations are significant, the tank model assumption of uniformity breaks down, and the 1D model and the tank model are expected to deviate. In fact, the tank predictions become sensitive to the delta chosen. As delta is increased, the apparent transport limitations increase in the tank model, resulting in decreased capacity and increased polarization. Generally, the tank model matches best with a delta between $1 / 2$ and $1 / 3$, depending on the parameters and rates being used. With the diffusion coefficient of $1 \times 10^{-12} \mathrm{~m}^{2} / \mathrm{s}$, the simulations are limited to the $0.2 \mathrm{C}$ rate and base case cathode thickness; higher rates and cathode thicknesses cause the tank model to experience a singularity.

When considering thicker cathodes, the trends are similar to the impact of lowering diffusion coefficients. Figure 4(a) shows the voltage curves of the tank and 1D model with double the cathode thickness $\left(80 \mu \mathrm{m}\right.$ and $1 \times 10^{-11} \mathrm{~m}^{2} / \mathrm{s}$ diffusion coefficient at $1 \mathrm{C}$ rate $)$. The 
diffusion limitations are increased when compared to the base case of $40 \mu \mathrm{m}$. As transport becomes more limiting, some detailed features of the discharge curve are missed in the tank model, such as the voltage dip in the 1D model, and the tank model somewhat overpredicts the capacity.

The power of physics-based models is the ability to identify underlying physical phenomena that govern the overall battery's voltage response. To illustrate this point, the temporal progression of partial currents for each charge transfer reaction (Eqs. 2-6) within the cathode are shown in Figure 4 (b) for the tank model and the 1D model, where the values are normalized by the applied current (set by C-rate) so they add to 1 at all times. For the 1D model, the partial currents are integrated across the cathode to obtain average values. Figure 4 (b) shows that the beginning of discharge is dominated by charge transfer through reaction (2) with some contributions from reaction (3), the next reaction in the reduction cascade. Small discrepancies between the $1 \mathrm{D}$ and tank model begin with the peak partial current from reaction (4), around the voltage transition between the two plateaus. The second voltage plateau is primarily set by the passage of current through the last two reactions, until the end of discharge. Figure 4 (c) shows the concentrations of all the ionic species within the cathode. Over different orders of magnitude, the models match well, and the shapes of the concentrations are captured well by the tank model even at these conditions. The volume fractions of the solid species are shown in Figure 4 (d). The tank model matches the $1 \mathrm{D}$ model well with a slight discrepancy at the beginning of the $\mathrm{Li}_{2} \mathrm{~S}$ precipitation, corresponding to the beginning of the second plateau in the voltage curve.

The solution for the tank model is faster and more computationally efficient, has fewer numerical instabilities, and does not require discretization along the cell thickness. However, there is a key dilemma in using a simplified model ideally suited to a limiting case (such as 
modest transport limitations), namely, are there short cut methods that can help us have confidence in a simplified model output, without needing to run the $1 \mathrm{D}$ model? To quantify the 1D model and tank model prediction accuracy, model variance was calculated in two different ways.

The first type of error calculation was the root mean square error (RMSE) of the voltage responses between different pairs of model predictions. In Figure 5 (a), the y axis represents the error between the 1D model and the tank model with delta of $1 / 2$ while the $x$ axis of Figure 5 (a) is the difference between two instances of the tank model with delta of $1 / 2$ and $1 / 3$. Recall delta represents the fraction of each region where gradients are expected. As shown in Figure 3, the tank model is sensitive to delta and deviates from the 1D model when transport limitations matter. Figure 5 (a) shows two ways to probe transport errors, by the RMSE error of the voltage measures. For every simulation performed, the variables being modified from base conditions are represented by color (diffusion), symbol shape (C-rate), and electrode thickness (light or dark shade). The majority of the points lie near or below around the 45-degree line, indicating the error between the 1D model and the tank is equal to or less than the error between the two tank models with delta varied. Fundamentally, this means that changing delta in the tank model is typically a more sensitive probe of whether the tank model assumptions are breaking down than a comparison of the tank to $1 \mathrm{D}$ model. Thus, it is generally not needed to use the 1D model to assess if transport is increasingly important. It is important to note that the RMSE for all simulations shown are below $25 \mathrm{mV}$ so, for the parameter ranges explored here, the tank model does quite well predicting the voltage response.

While the RMSE captures deviations in voltage between the two models, the second type of error emphasizes the total capacity, another important feature. In Figure 5 (b), the normalized 
capacity errors between the models are plotted, with the differences between the 1D model and tank model on the $\mathrm{y}$ axis and differences between the two deltas in tank models on the $\mathrm{x}$ axis. To make these calculations, every final capacity value is divided by the theoretical capacity. On average, the capacity errors lie in an envelope below the 45-degree line, again indicating better agreement between the 1D model and the tank model with delta $1 / 2$ than between the tank models with deltas of $1 / 2$ and $1 / 3$. The same general trends related to the effects of diffusion coefficients, C-rates, and cathode thicknesses are seen in Fig. 5a and Fig. 5b, with more clustering by parameter within the capacity errors. Importantly, the errors in capacity are less than $7 \%$, for all simulations considered.

To illustrate the relevance of both the 1D and the tank model for experiments, Figure 6 (a) shows a C/5 discharge curve from the proprietary lithium sulfur pouch cell. In Figure 6 (b), the tank and the 1D model predictions are compared at $\mathrm{C} / 5$. The overall features and shape of the experiment curve, including the characteristic two plateaus, are captured well by both the tank and the 1D model. The agreement between the two models and experiment in Fig. 6 suggests we are working with a reasonable basis set of parameters. Future work will present detailed discussion on parameter estimation and validation at different conditions and rates for lithium sulfur batteries. 


\section{Conclusions and Implications}

In this work, we have discussed the development of a tank-in-series model that is volume averaged from the 1D lithium sulfur mathematical model for discharge ${ }^{3}$. The tank captures overall trends and average variables in a lithium sulfur battery. Although it is a lumped model, the diffusion coefficients play a role in transport between the two tanks, in a similar fashion to the detailed 1D, spatially resolved model. For the parameter set that is considered within this work, the tank model predictions match well in the majority of simulations and provide the features predicted by the 1D model. The voltage shape is matched very well while the capacity is more variable in diffusion-affected regimes.

While the one-dimensional model contains more information than the tank-in-series model, extra information comes at a computational cost as the 1D model takes 1-2 orders of magnitude longer to run than the tank model. The increased computational speed and efficiency make the tank model an ideal candidate for parameter estimation, where it can be leveraged to identify regions where the model matches experimental data well. If further spatial resolution is vital to understanding a problem, the initial guesses from the tank model can then be fed into the 1D model because the parameters match one-to-one. While other lumped models focus on trend predictions and speed, the tank model can qualitatively match experimental data with increased speed, compared to the 1D model.

Based on an analysis of errors in Figure 6, it is recommended users implement the tank model with delta as $1 / 2$ for parameter estimation or design. When parameters are found, compare the simulations between the tank models with delta of $1 / 2$ and a second calculation with delta of $1 / 3$ (and all other parameters set). If there is an acceptable voltage RMSE or capacity 
difference between the two tank simulations, then transport limitations are deemed modest, and the tank model is expected to be an acceptable representation of the 1D model. If the error between the two tank models (with delta of $1 / 2$ and 1/3) is unacceptably large, the tank results can be deemed a first guess for a 1D model estimation or design analysis.

As modeling of lithium sulfur batteries advances and mechanisms are improved, the tankin-series model can be modified easily to include more physics as needed. For future studies, the focus will be on using the tank-in-series model in parameter estimation to improve model fit to experimental data at for a range of rates and conditions.

\section{Acknowledgments}

The authors would like to thank the U.S. Department of Energy (DOE) for providing partial financial support for this work, through the Advanced Research Projects Agency (ARPA-E) award number DE-AR0000275, Advanced Battery Material Research (BMR) Program (Battery 500 Consortium). The authors would also like to thank the Clean Energy Institute (CEI) at the University of Washington, and Texas Materials Institute for partial monetary support of this work.

\section{List of Symbols}

\begin{tabular}{|l|l|}
\hline$A$ & Specific surface area of cathode, $\mathrm{m}^{2} / \mathrm{m}^{3}$ \\
\hline$a_{0}$ & Initial value of $a, \mathrm{~m}^{2} / \mathrm{m}^{3}$ \\
\hline$B$ & Bruggeman coefficient \\
\hline$C_{l, i}$ & Concentration of species $i, \mathrm{~mol} / \mathrm{m}^{3}$ \\
\hline
\end{tabular}




\begin{tabular}{|c|c|}
\hline$C_{i, r e f}$ & Reference concentration of species $i, \mathrm{~mol} / \mathrm{m}^{3}$ \\
\hline$D_{i, 0}$ & $\begin{array}{l}\text { Diffusion coefficient of species } \text { i in the bulk } \\
\text { medium, } \mathrm{m}^{2} / \mathrm{s}\end{array}$ \\
\hline$D_{i}$ & $\begin{array}{l}\text { Diffusion coefficient of species } i \text { in the porous } \\
\text { medium } \mathrm{m}^{2} / \mathrm{s}\end{array}$ \\
\hline$F$ & Faraday constant, $\mathrm{C} / \mathrm{mol}$ \\
\hline$i_{j}$ & Current density from reaction $j, A / m^{2}$ \\
\hline$i_{0, \text { rref }}$ & $\begin{array}{l}\text { Exchange current density of reaction } j \text { at } \\
\text { reference concentrations, } A / m^{2}\end{array}$ \\
\hline$i_{e}$ & $\begin{array}{l}\text { Superficial current density in the electrolyte } \\
\text { phase, } A / m^{2}\end{array}$ \\
\hline$i_{s}$ & $\begin{array}{l}\text { Superficial current density in the solid phase, } \\
A / m^{2}\end{array}$ \\
\hline$I_{a p p}$ & Applied current density, $A / m^{2}$ \\
\hline$K_{s p, k}$ & $\begin{array}{l}\text { Solubility product of precipitate } k \text {, varying } \\
\text { units, see Table } V\end{array}$ \\
\hline$k_{k}$ & $\begin{array}{l}\text { Rate constant of precipitate } k \text {, varying units, } \\
\text { see Table V }\end{array}$ \\
\hline$l_{1}$ & Thickness of the cathode, $m$ \\
\hline$l_{2}$ & Thickness of the separator, $m$ \\
\hline$N_{l, i}$ & Superficial flux of species $i$ in region $l, \mathrm{~mol}$ \\
\hline
\end{tabular}




\begin{tabular}{|c|c|}
\hline & $m 2 s^{-1}$ \\
\hline$n_{j}$ & $\begin{array}{l}\text { Number of electrons transferred in } \\
\text { electrochemical reaction } j\end{array}$ \\
\hline$R$ & Gas constant, $\mathrm{J} \mathrm{mol}^{-1} \mathrm{~K}^{-1}$ \\
\hline$R_{l, i}$ & $\begin{array}{l}\text { Production rate of species } i \text { due to } \\
\text { precipitation reactions in region l, } \mathrm{mol} \mathrm{m}^{3} \mathrm{~s}^{-1}\end{array}$ \\
\hline$R_{k}^{\prime}$ & $\begin{array}{l}\text { Rate of precipitation of solid species } k, \mathrm{~mol} \\
\mathrm{~m}^{3} \mathrm{~s}^{-1}\end{array}$ \\
\hline$r_{i}$ & $\begin{array}{l}\text { Production rate of species i from } \\
\text { electrochemical reactions, } \text { mol m}^{3} \mathrm{~s}^{-1}\end{array}$ \\
\hline$s_{a, j}$ & $\begin{array}{l}\text { Stoichiometric coefficient of anodic species in } \\
\text { electrochemical } j\end{array}$ \\
\hline$s_{c, j}$ & $\begin{array}{l}\text { Stoichiometric coefficient of cathodic species } \\
\text { in electrochemical } j\end{array}$ \\
\hline$s_{i, j}$ & $\begin{array}{l}\text { Stoichiometric coefficient of species I in } \\
\text { electrochemical reaction } j\end{array}$ \\
\hline$T$ & Temperature, $K$ \\
\hline$T$ & Time, $s$ \\
\hline$U_{j}^{\theta}$ & $\begin{array}{l}\text { Standard OCP of electrochemical reaction } j, \\
V\end{array}$ \\
\hline$U_{j, r e f}$ & $\begin{array}{l}\text { OCP of electrochemical reaction } j \text { at } \\
\text { reference concentrations, } V\end{array}$ \\
\hline
\end{tabular}




\begin{tabular}{|l|l|}
\hline$\tilde{V}_{k}$ & Molar volume of the precipitate $\mathrm{k}, \mathrm{m}^{3} / \mathrm{mol}$ \\
\hline$z_{i}$ & Charge number of species $i$ \\
\hline
\end{tabular}

Greek symbols

\begin{tabular}{|c|c|}
\hline$\alpha_{a j}$ & Anodic transfer coefficient of reaction $j$ \\
\hline$\alpha_{c j}$ & Cathodic transfer coefficient of reaction $j$ \\
\hline$\delta$ & $\begin{array}{l}\text { Fraction of domain where gradients are } \\
\text { assumed to be present }\end{array}$ \\
\hline$\delta_{l}$ & $\begin{array}{l}\text { 'Diffusion length' approximation, which is } \\
\text { some fraction of the region } l, m\end{array}$ \\
\hline$\gamma_{i, k}$ & $\begin{array}{l}\text { Number of ionic species i produced by the } \\
\text { dissociation of precipitate } k\end{array}$ \\
\hline$\varepsilon_{l}$ & Porosity of region $l$ \\
\hline$\overline{\varepsilon_{l, k}}$ & Volume fraction of precipitate $k$ in region $l$ \\
\hline$\eta_{j}$ & Overpotential for electrochemical reaction $j$ \\
\hline$\xi$ & Morphology parameter \\
\hline$\sigma$ & $\begin{array}{l}\text { Effective conductivity of solid phase of the } \\
\text { cathode, } S / m\end{array}$ \\
\hline$\phi_{l, e}$ & Potential in the liquid phase in region $l, V$ \\
\hline$\phi_{s}$ & Potential in the solid phase in region $l, V$ \\
\hline
\end{tabular}




\section{References}

1. T. Cleaver, P. Kovacik, M. Marinescu, T. Zhang, and G. Offer, J. Electrochem. Soc., 165, A6029-A6033 (2018).

2. Y. V. Mikhaylik and J. R. Akridge, J. Electrochem. Soc., 151, A1969 - A1976 (2004).

3. K. Kumaresan, Y. Mikhaylik, and R. E. White, J. Electrochem. Soc., 155, A576 - A582 (2008).

4. J. P. Neidhardt et al., J. Electrochem. Soc., 159, A1528-A1542 (2012).

5. D. N. Fronczek and W. G. Bessler, J. Power Sources, 244, 183-188 (2013).

6. K. Yoo, M. K. Song, E. J. Cairns, and P. Dutta, Electrochim. Acta, 213, 174-185 (2016).

7. A. F. Hofmann, D. N. Fronczek, and W. G. Bessler, J. Power Sources, 259, 300-310 (2014).

8. M. Marinescu et al., J. Electrochem. Soc., 165, A6107-A6118 (2018).

9. T. Zhang, M. Marinescu, S. Walus, and G. J. Offer, Electrochim. Acta, 219, 502-508 (2016).

10. M. Marinescu, T. Zhang, and G. J. Offer, Phys. Chem. Chem. Phys., 18, 584-593 (2016).

11. N. Erisen, N. B. Emerce, S. C. Erensoy, and D. Eroglu, Int. J. Energy Res., 42, 2631-2642 (2018).

12. C. Michaelis, N. Erisen, D. Eroglu, and G. M. Koenig, Int. J. Energy Res., 43, 874-883 (2018).

13. T. Zhang, M. Marinescu, L. O’Neill, M. Wild, and G. Offer, Phys. Chem. Chem. Phys., 17, 22581-22586 (2015).

14. Y. X. Ren, T. S. Zhao, M. Liu, P. Tan, and Y. K. Zeng, J. Power Sources, 336, 115-125 (2016).

15. M. Marinescu, T. Zhang, and G. J. Offer, Phys. Chem. Chem. Phys., 18, 584-593 (2016). 
16. N. B. Emerce and D. Eroglu, J. Electrochem. Soc., 166, A1490-A1500 (2019).

17. M. Safari, C. Y. Kwok, and L. F. Nazar, ACS Cent. Sci., 2, 560-568 (2016).

18. V. Ramadesigan et al., J. Electrochem. Soc., 159, R31-R45 (2012).

19. S. Walü̈ et al., Adv. Energy Mater., 5, 1-5 (2015).

20. A. Subramaniam et al., J. Electrochem. Soc., 167, 013534 (2020).

21. M. T. Lawder, V. Ramadesigan, B. Suthar, and V. R. Subramanian, Comput. Chem. Eng., 82, 283-292 (2015).

22. A. Tomaszewska et al., eTransportation, 1, 100011 (2019).

23. K. S. Han et al., Chem. Mater., 29, 9023-9029 (2017). 


\section{List of Figures}

Figure 1. (a) Physical representation of the lithium sulfur battery, left to right: copper current collector, cathode with thickness of $l_{1}$ filled with yellow $S_{8(s)}$ particles and conductive carbon additives with electrolyte, porous separator in blue with thickness of $l_{2}$, and the lithium foil

anode, (b) Schematic of the tank-in-series model that volume averages the cathode and separator of corresponding thicknesses of $l_{1}$ and $l_{2}$, resulting in average variables in each tank. Mass flow between the tanks is connected through interfacial fluxes.

Figure 2. Comparison of tank-in-series model $(\delta=1 / 3)$ and $1 \mathrm{D}$ model predictions at rates of $0.2 \mathrm{C}$ (blue), $0.5 \mathrm{C}$ (green), and $1 \mathrm{C}$ (orange) (a) with diffusion coefficient of $1 \times 10^{-10} \mathrm{~m}^{2} / \mathrm{s}$ where results are almost identical, (b) with diffusion coefficient of $1 \times 10^{-11} \mathrm{~m}^{2} / \mathrm{s}$ where transport limitations are introduced and some variation is introduced at the end of discharge.

Figure 3. Effect of the parameter delta on the tank predictions at $0.2 \mathrm{C}$ with diffusion coefficient of $1 \times 10^{-12} \mathrm{~m}^{2} / \mathrm{s}$.

Figure 4. Predictions for the $1 \times 10^{-11} \mathrm{~m}^{2} / \mathrm{s}$ diffusion constant with thickness double the base case at a rate of $1 \mathrm{C}$ with the tank model $(\delta=1 / 2)$ and the $1 \mathrm{D}$ model (a) voltage curves with some deviation between the two models during the initial drop, transition to the second plateau, and end of discharge; (b) the partial currents of the electrochemical Reactions (2) through (6) of the sulfur cascade, which have been normalized by the overall applied current, $I_{a p p}$; (c) logarithmic plot of the concentration of ionic species within the cathode throughout discharge, (d) plot of the volume fractions of the species, $\mathrm{S}_{8(\mathrm{~s})}$ and $\mathrm{Li}_{2} \mathrm{~S}_{(\mathrm{s})}$. In Figure 5(b)-(d), the values for the 1D model have been integrated over the cathode domain to compare to the average values of the tank model. 
Figure 5. Plots of the (a) of the root mean square error (RMSE) of the voltage, (b) of the total capacity normalized by the theoretical capacity error where the y axis is the error between the 1D model and the tank model $(\delta=1 / 2)$ and the $\mathrm{x}$ axis denotes the error between two tank model simulations $(\delta=1 / 2,1 / 3)$. Each of the symbol and color combinations represents a different set of levels for each parameter, denoted by color (diffusion coefficient), symbol (rate), and shade (cathode thickness).

Figure 6. Lithium Sulfur Discharge Curves (a) from experimental data of a proprietary, precommercial pouch cell at $\mathrm{C} / 5$, (b) simulated curves comparing the $1 \mathrm{D}$ model and the tank-inseries model at $\mathrm{C} / 5$ with estimated parameters that exhibit the all the main features from the experimental curve. 
a

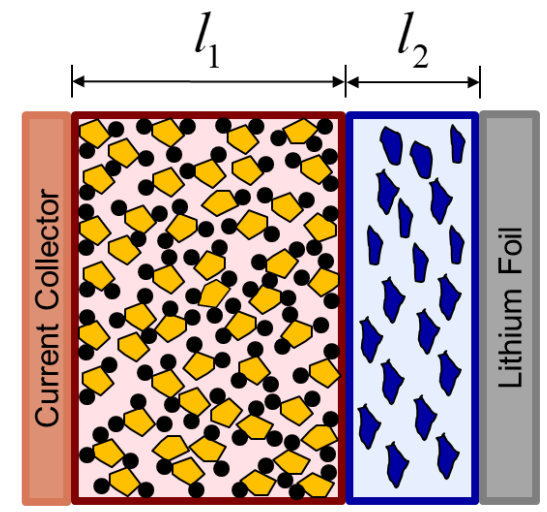

b

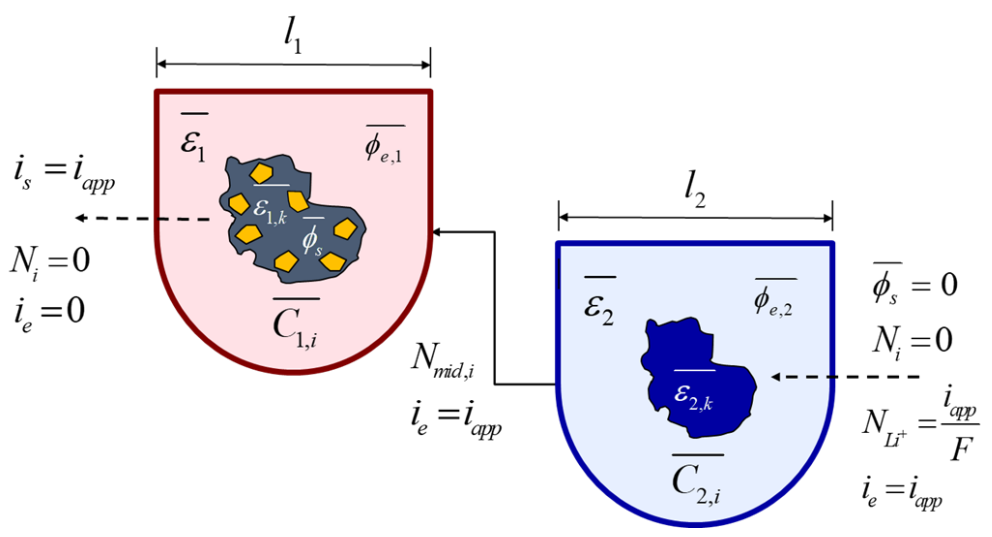

Figure 1. (a) Physical representation of the lithium sulfur battery, left to right: copper current collector, cathode with thickness of $\boldsymbol{l}_{1}$ filled with yellow $\boldsymbol{S}_{\boldsymbol{8}(s)}$ particles and conductive carbon additives with electrolyte, porous separator in blue with thickness of $l_{2}$, and the lithium foil anode, (b) Schematic of the tank-in-series model that volume averages the cathode and separator of corresponding thicknesses of $l_{1}$ and $l_{2}$, resulting in average variables in each tank. Mass flow between the tanks is connected through interfacial fluxes. 

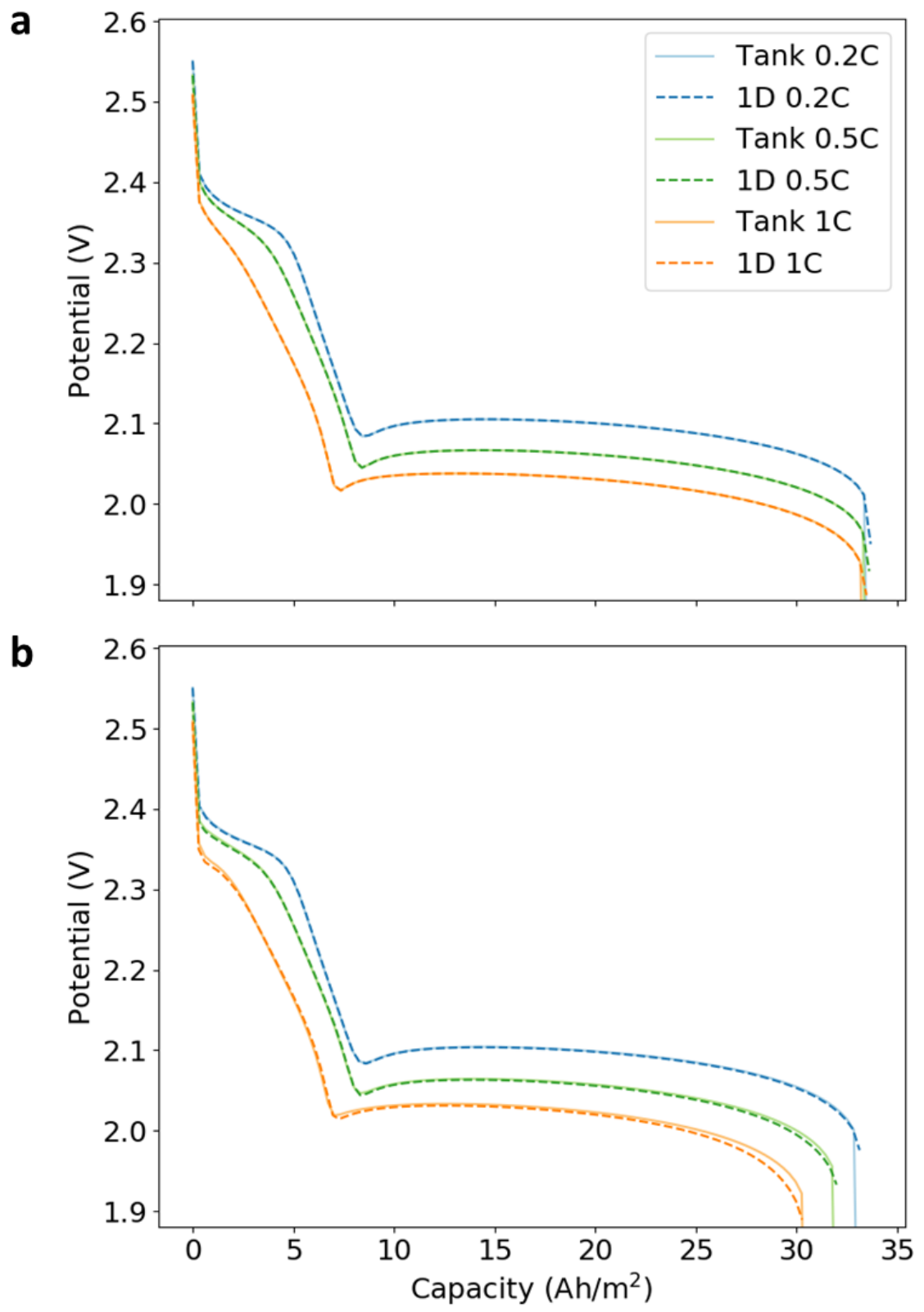

Figure 2. Comparison of tank-in-series model $(\delta=1 / 3)$ and $1 \mathrm{D}$ model predictions at rates of 0.2C (blue), $0.5 \mathrm{C}$ (green), and 1C (orange) (a) with diffusion coefficient of $1 \times 10^{-10} \mathrm{~m}^{2} / \mathrm{s}$ where results are almost identical, (b) with diffusion coefficient of $1 \times 10^{-11} \mathrm{~m}^{2} / \mathrm{s}$ where transport limitations are introduced and some variation is introduced at the end of discharge. 


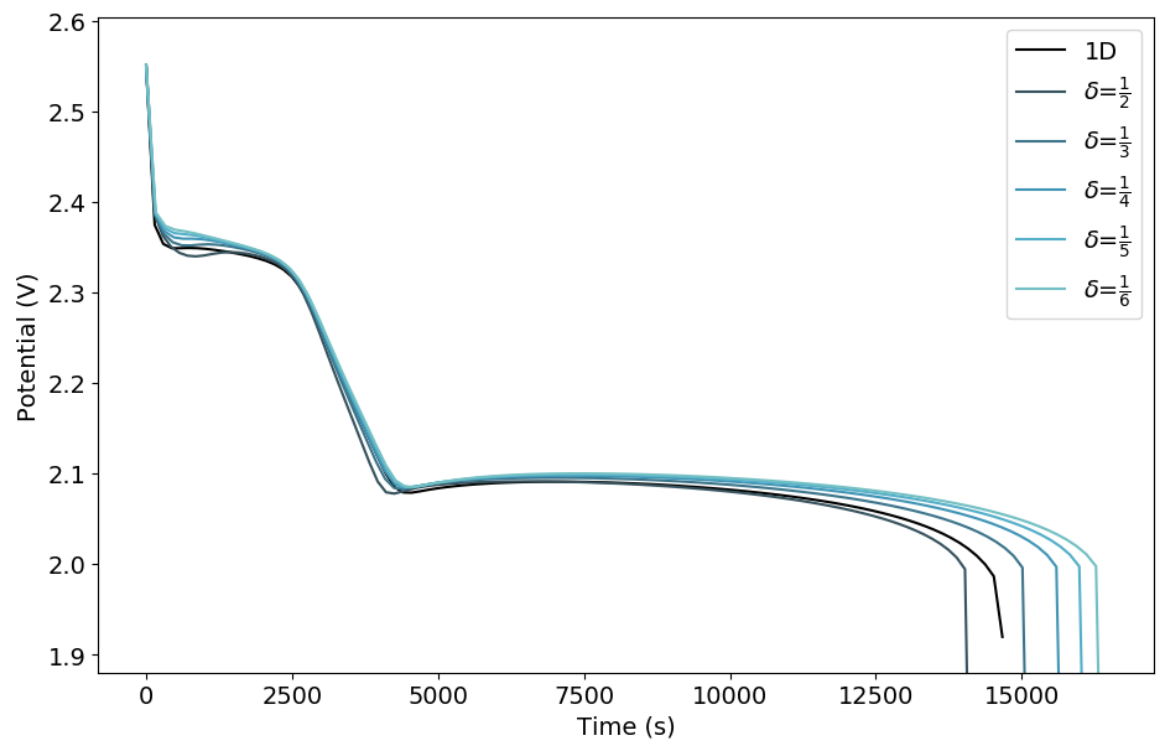

Figure 3. Effect of the parameter delta on the tank predictions at $0.2 \mathrm{C}$ with diffusion coefficient of $1 \times 10^{-12} \mathrm{~m}^{2} / \mathrm{s}$. 

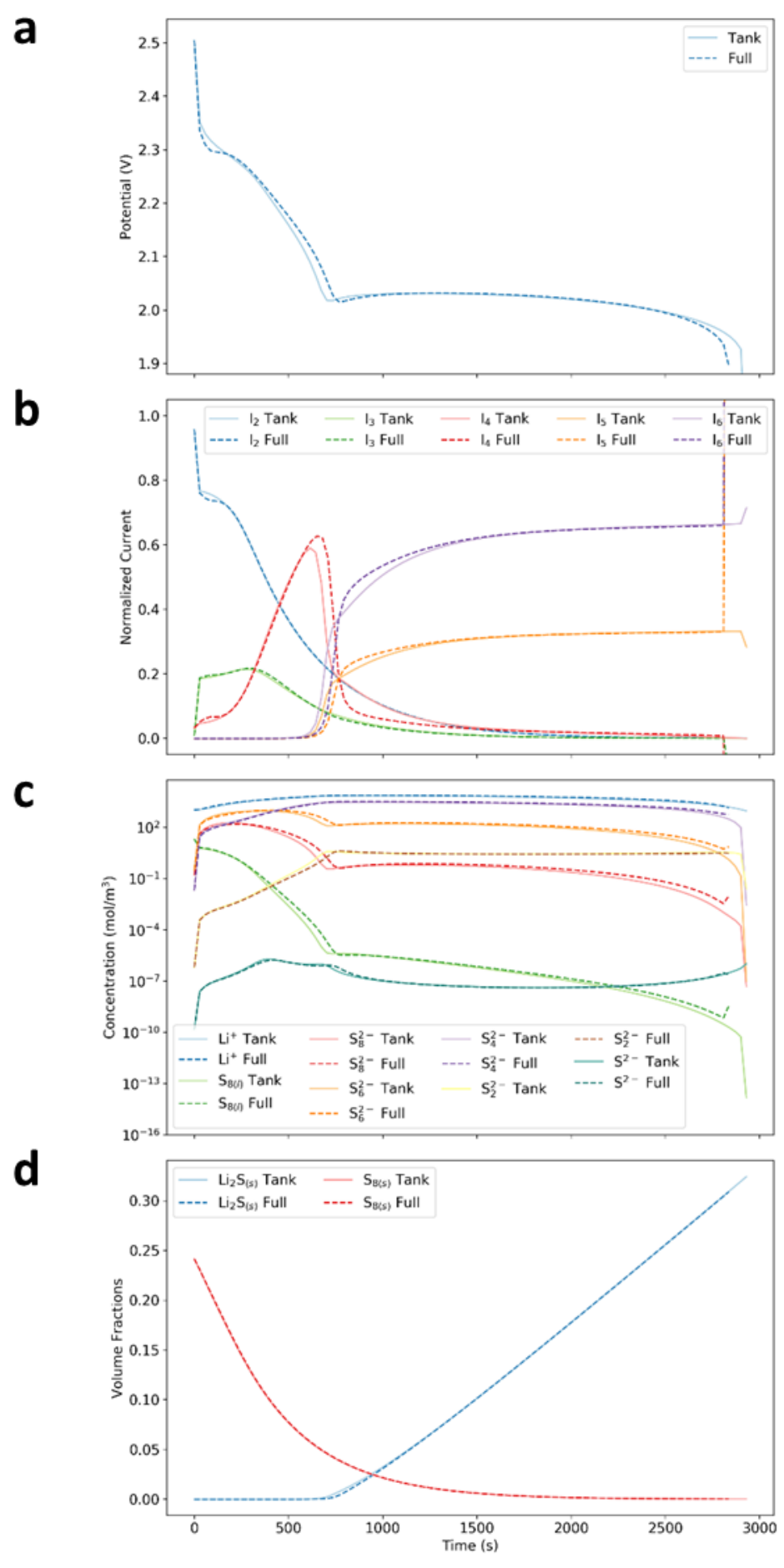

Figure 4. Predictions for the $1 \times 10^{-11} \mathrm{~m}^{2} / \mathrm{s}$ diffusion constant with thickness double the base case at a rate of $1 \mathrm{C}$ with the tank model $(\delta=1 / 2)$ and the $1 \mathrm{D}$ model (a) voltage curves with some deviation between the two models during the initial drop, transition to the second plateau, and end of discharge; (b) the partial currents of the electrochemical Reactions (2) 
through (6) of the sulfur cascade, which have been normalized by the overall applied current, $I_{a p p}$; (c) logarithmic plot of the concentration of ionic species within the cathode throughout discharge, (d) plot of the volume fractions of the species, $\mathbf{S}_{8(\mathrm{~s})}$ and $\mathbf{L i}_{2} \mathbf{S}_{(\mathrm{s})}$. In Figure 5(b)-(d), the values for the 1D model have been integrated over the cathode domain to compare to the average values of the tank model. 


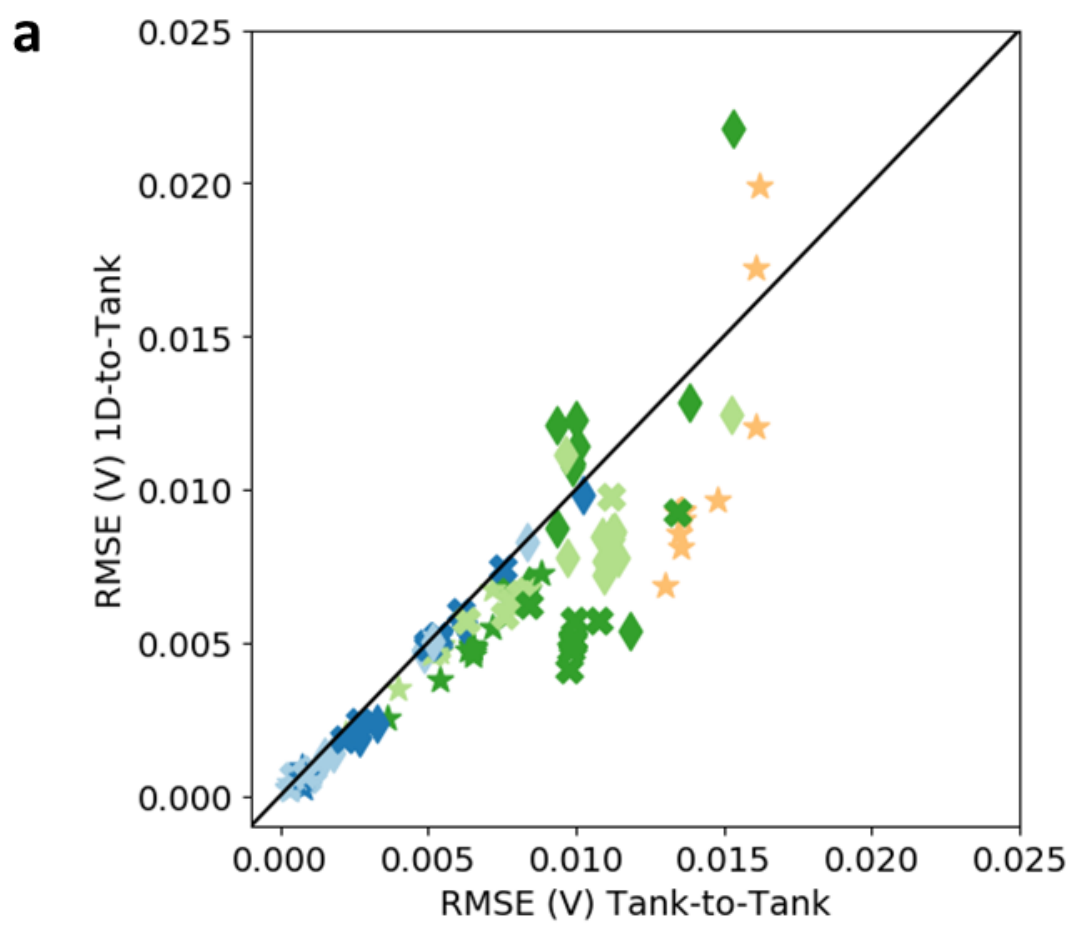

b

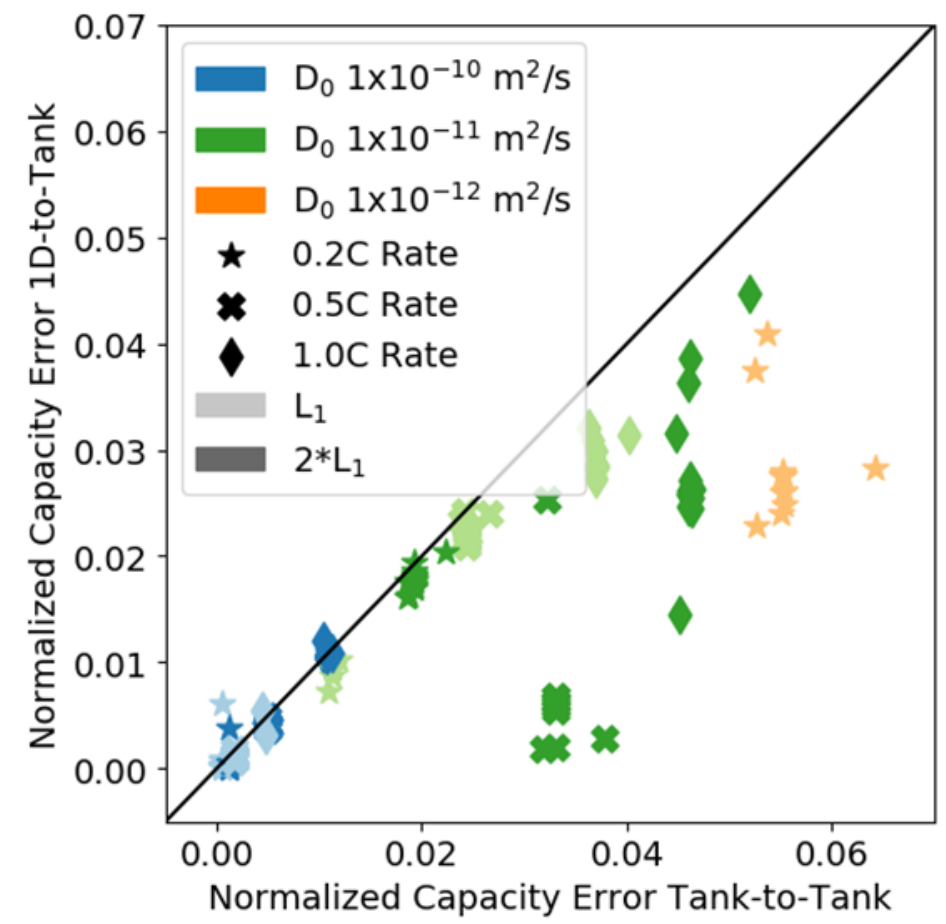

Figure 5. Plots of the (a) of the root mean square error (RMSE) of the voltage, (b) of the total capacity normalized by the theoretical capacity error where the $y$ axis is the error between the 1D model and the tank model $(\delta=1 / 2)$ and the $\mathbf{x}$ axis denotes the error between two tank model simulations $(\delta=1 / 2,1 / 3)$. Each of the symbol and color combinations 
represents a different set of levels for each parameter, denoted by color (diffusion coefficient), symbol (rate), and shade (cathode thickness). 

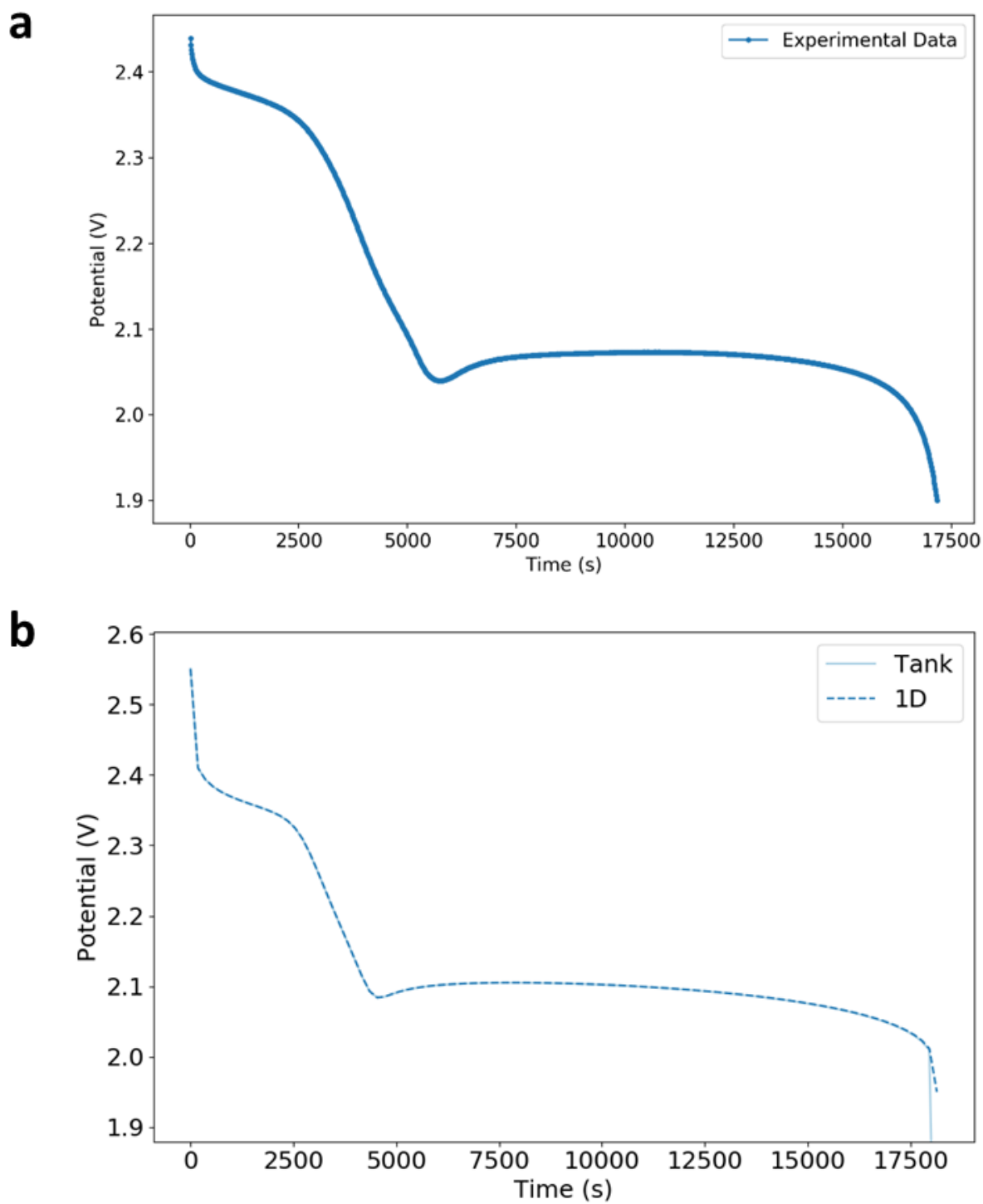

Figure 6. Lithium Sulfur Discharge Curves (a) from experimental data of a proprietary, pre-commercial pouch cell at $\mathrm{C} / 5$, (b) simulated curves comparing the $1 \mathrm{D}$ model and the tank-in-series model at $\mathrm{C} / 5$ with estimated parameters that exhibit the all the main features from the experimental curve. 


\section{List of Tables}

Table I. 1-D Lithium Sulfur Mathematical Discharge Model.

Table II. Cathode and Separator Parameters.

Table III. Parameters for the Ionic Species.

Table IV. Properties of the Electrochemical Reactions.

Table V. Parameters for the precipitation reactions.

Table VI. Equations for the Tank-in-Series Model for Lithium Sulfur Batteries.

Table VII. Equations of the Tank-in-Series Model with Logarithmic Time Scaling.

Table VIII. Values of the Parameter Study. 


\section{Table I. 1-D Lithium Sulfur Mathematical Discharge Model.}

\section{Governing Equations}

Boundary

\section{Conditions}

\begin{tabular}{|c|c|}
\hline Positive Electrode (Region 1) & \\
\hline$\frac{\partial \varepsilon_{1} C_{1, i}}{\partial t}=-\frac{\partial N_{1, i}}{\partial x}+r_{i}-R_{1, i}$ & $\left.C_{1, i}\right|_{x=l_{1}}=\left.C_{2, i}\right|_{x=l_{1}}$ \\
\hline$\frac{N_{1, i}}{\varepsilon_{1}}=-D_{1, i} \frac{\partial C_{1, i}}{\partial x}-z_{i} \frac{D_{1, i}}{R T} F C_{1, i} \frac{\partial \phi_{1, e}}{\partial x}$ & $\left.N_{1, i}\right|_{x=0}=0$ \\
\hline$i_{s}=-\sigma \frac{\partial \phi_{s}}{\partial x}$ & $-\left.\sigma \frac{\partial \phi_{s}}{\partial x}\right|_{x=0}=I_{a p p}$ \\
\hline$i_{1, e}=F \sum_{i} z_{i} N_{1, i}$ & $\left.i_{1, e}\right|_{x=0}=0$ \\
\hline$\frac{\partial \varepsilon_{1, k}}{\partial t}=\tilde{V}_{k} R_{k}^{\prime}$ & $\left.N_{1, i}\right|_{x=l_{1}}=\left.N_{2, i}\right|_{x=l_{1}}$ \\
\hline$\frac{\partial \varepsilon_{1}}{\partial t}=-\sum_{k} \tilde{V}_{k} R_{k}^{\prime}$ & $-\left.\sigma \frac{\partial \phi_{s}}{\partial x}\right|_{x=l_{1}}=0$ \\
\hline Separator (Region 2) & \\
\hline$\frac{\partial \varepsilon_{2} C_{2, i}}{\partial t}=-\frac{\partial N_{2, i}}{\partial x}-R_{2, i}$ & $\left.N_{1, i}\right|_{x=l_{1}+l_{2}}=0$ \\
\hline$\frac{N_{2, i}}{\varepsilon_{1}}=-D_{2, i} \frac{\partial C_{2, i}}{\partial x}-z_{i} \frac{D_{2, i}}{R T} F C_{2, i} \frac{\partial \phi_{2, e}}{\partial x}$ & $\left.N_{2, L i^{+}}\right|_{x=l_{1}+l_{2}}=\frac{I_{a p p}}{F}$ \\
\hline$i_{2, e}=F \sum_{i} z_{i} N_{2, i}$ & $\left.i_{1, e}\right|_{x=l_{1}}=\left.i_{2, e}\right|_{x=l_{1}}$ \\
\hline$\frac{\partial \varepsilon_{2, k}}{\partial t}=\tilde{V}_{k} R_{k}^{\prime}$ & $\left.\phi_{s}\right|_{x=l_{1}+l_{2}}=0$ \\
\hline$\frac{\partial \varepsilon_{2}}{\partial t}=-\sum_{k} \tilde{V}_{k} R_{k}^{\prime}$ & $\left.i_{2, e}\right|_{x=l_{1}+l_{2}}=F N_{L i^{+}}$ \\
\hline
\end{tabular}

\section{Other Expressions}

$r_{i}=-a \sum_{j} \frac{s_{i, j} i_{j}}{n_{j} F}$

$a=a_{0}\left(\frac{\varepsilon}{\varepsilon_{\text {initial }}}\right)^{\xi}$

$i_{j}=i_{o, j_{\text {ref }}}\left\{\left(\frac{C_{i, \text { anodic }}}{C_{i, \text { ref }}}\right)^{s_{a, j}} \exp \left(\frac{\alpha_{a, j} F}{R T} \eta_{j}\right)-\left(\frac{C_{i, \text { cathodic }}}{C_{i, \text { ref }}}\right)^{-s_{c, j}} \exp \left(-\frac{\alpha_{c, j} F}{R T} \eta_{j}\right)\right\}$

$\eta_{j}=\phi_{s}-\phi_{e}-U_{j, r e f}$ 


$$
\begin{aligned}
& U_{j, r e f}=U_{j}^{\theta}-\frac{R T}{n_{j} F} \sum_{i} s_{i j} \ln \left[\frac{C_{i}}{1000}\right] \\
& \frac{\partial i_{s}}{\partial x}+\frac{\partial i_{e}}{\partial x}=0 \\
& \frac{\partial i_{e}}{\partial x}=a \sum_{j} i_{j} \\
& R_{k}^{\prime}=k_{k} \varepsilon_{1, k}\left(\prod_{i} C_{1, i}^{\gamma_{i, k}}-K_{s p, k}\right) \\
& R_{i}=\sum_{k} \gamma_{i, k} R_{k}^{\prime}
\end{aligned}
$$

\begin{tabular}{ccc}
$\begin{array}{c}\text { Table II. Cathode and Separator Parameters. } \\
\text { Parameter }\end{array}$ & $\begin{array}{c}\text { Cathode } \\
\text { Separator }\end{array}$ \\
\hline Thickness $(\mu \mathrm{m})^{\mathrm{a}}$ & $20^{\mathrm{b}}$ \\
$a_{0}\left(\mathrm{~m}^{2} / \mathrm{m}^{3}\right)^{\mathrm{a}}$ & 143292 & - \\
$\varepsilon_{\text {initial }}{ }^{\mathrm{a}}$ & 0.54 & 0.6 \\
$\varepsilon_{S_{8(s)}, \text { initial }}$ & $0.24^{\mathrm{c}}$ & $1 \times 10^{-12 \mathrm{~b}}$ \\
$\varepsilon_{L_{2} S_{(s)}, \text { initial }^{\mathrm{b}}}$ & $1 \times 10^{-7}$ & $1 \times 10^{-7}$ \\
$\sigma^{\mathrm{a}}$ & 937 & - \\
$\xi^{\mathrm{b}}$ & 1.5 & - \\
$T_{(K)^{\mathrm{b}}}$ & 293 & 293 \\
Bruggeman coefficient & 2.5 & 2.5 \\
\hline
\end{tabular}

${ }^{\mathrm{a}}$ estimated parameters, ${ }^{\mathrm{b}}$ taken from Kumaresan, ${ }^{\mathrm{c}}$ design parameters

Table III. Parameters for the Ionic Species.

\begin{tabular}{cccc} 
Species $(\mathbf{i})$ & $z_{i}$ & $D_{i 0}\left(\mathbf{m}^{2} / \mathbf{s}\right)$ & $C_{i, r e f}\left(\mathbf{m o l} / \mathbf{m}^{3}\right)$ \\
\hline$L i^{+}$ & +1 & $1 \times 10^{-10 \mathrm{c}}$ & $1033^{\mathrm{a}}$ \\
$S_{8(l)}$ & 0 & $1 \times 10^{-10 \mathrm{c}}$ & $19.9^{\mathrm{a}}$ \\
$S_{8}^{2-}$ & -2 & $1 \times 10^{-10 \mathrm{c}}$ & $0.16^{\mathrm{a}}$ \\
$S_{6}^{2-}$ & -2 & $1 \times 10^{-10 \mathrm{c}}$ & $0.31^{\mathrm{a}}$ \\
$S_{4}^{2-}$ & -2 & $1 \times 10^{-10 \mathrm{c}}$ & $0.020^{\mathrm{b}}$ \\
$S_{2}^{2-}$ & -2 & $1 \times 10^{-10 \mathrm{c}}$ & $0.56 \times 10^{-6 \mathrm{a}}$ \\
\hline
\end{tabular}




\begin{tabular}{cccc}
\hline$S^{2-}$ & -2 & $1 \times 10^{-10 \mathrm{c}}$ & $0.78 \times 10^{-9} \mathrm{a}$ \\
$A^{-}$ & -1 & $1 \times 10^{-9 \mathrm{a}, 23}$ & $1032^{\mathrm{a}}$ \\
\hline
\end{tabular}

${ }^{\mathrm{a}}$ estimated parameters, ${ }^{\mathrm{b}}$ taken from Kumaresan, ${ }^{\mathrm{c}}$ design parameters

Table IV. Properties of the Electrochemical Reactions.

\begin{tabular}{ccccccc} 
Reaction $(j)$ & $i_{0, j r e f}\left(\mathbf{A} / \mathbf{m}^{2}\right)^{\mathbf{c}}$ & $U_{j}^{\theta}(\mathbf{V})^{\mathbf{a}}$ & $s_{a, j}$ & $s_{c, j}$ & $\alpha_{a, j} \mathbf{b}$ & $\alpha_{c, j} \mathbf{b}$ \\
\hline $\mathbf{2}$ & 1.972 & 2.50 & $-1 / 2$ & $1 / 2$ & $1 / 2$ & $1 / 2$ \\
$\mathbf{3}$ & 0.019 & 2.49 & $-3 / 2$ & 2 & $1 / 2$ & $1 / 2$ \\
$\mathbf{4}$ & 0.019 & 2.42 & -1 & $3 / 2$ & $1 / 2$ & $1 / 2$ \\
$\mathbf{5}$ & $1.97 \times 10^{-4}$ & 2.12 & $-1 / 2$ & 1 & $1 / 2$ & $1 / 2$ \\
$\mathbf{6}$ & $1.97 \times 10^{-7}$ & 2.00 & $-1 / 2$ & 1 & $1 / 2$ & $1 / 2$ \\
\hline
\end{tabular}

${ }^{a}$ estimated parameters, ${ }^{b}$ taken from Kumaresan, ${ }^{c}$ design parameters

\begin{tabular}{|c|c|c|c|}
\hline $\begin{array}{c}\text { Precipitate } \\
(k)\end{array}$ & $\begin{array}{c}\text { Rate Constant } \\
\qquad\left(k_{k}\right)\end{array}$ & $\begin{array}{l}\text { Solubility Product } \\
\qquad\left(K_{k}\right)^{\mathbf{a}}\end{array}$ & $\begin{array}{c}\text { Molar Volume }\left(V_{k}\right) \\
\left(\mathbf{m}^{3} / \mathbf{m o l}\right)^{\mathbf{b}}\end{array}$ \\
\hline $\mathrm{S}_{8(\mathrm{~s})}$ & $1.0 \mathrm{~s}^{-1 \mathrm{~b}}$ & 20. $\mathrm{mol} \mathrm{m}^{-3}$ & $1.239 \times 10^{-4}$ \\
\hline $\mathrm{Li}_{2} \mathrm{~S}_{(\mathrm{s})}$ & 27. $\mathrm{m}^{6} \mathrm{~mol}^{2} \mathrm{~s}^{-1 \mathrm{a}}$ & $2.8 \times 10^{-5} \mathrm{~mol}^{3} \mathrm{~m}^{-9}$ & $2.768 \times 10^{-5}$ \\
\hline
\end{tabular}

${ }^{\mathrm{a}}$ estimated parameters, ${ }^{\mathrm{b}}$ taken from Kumaresan 


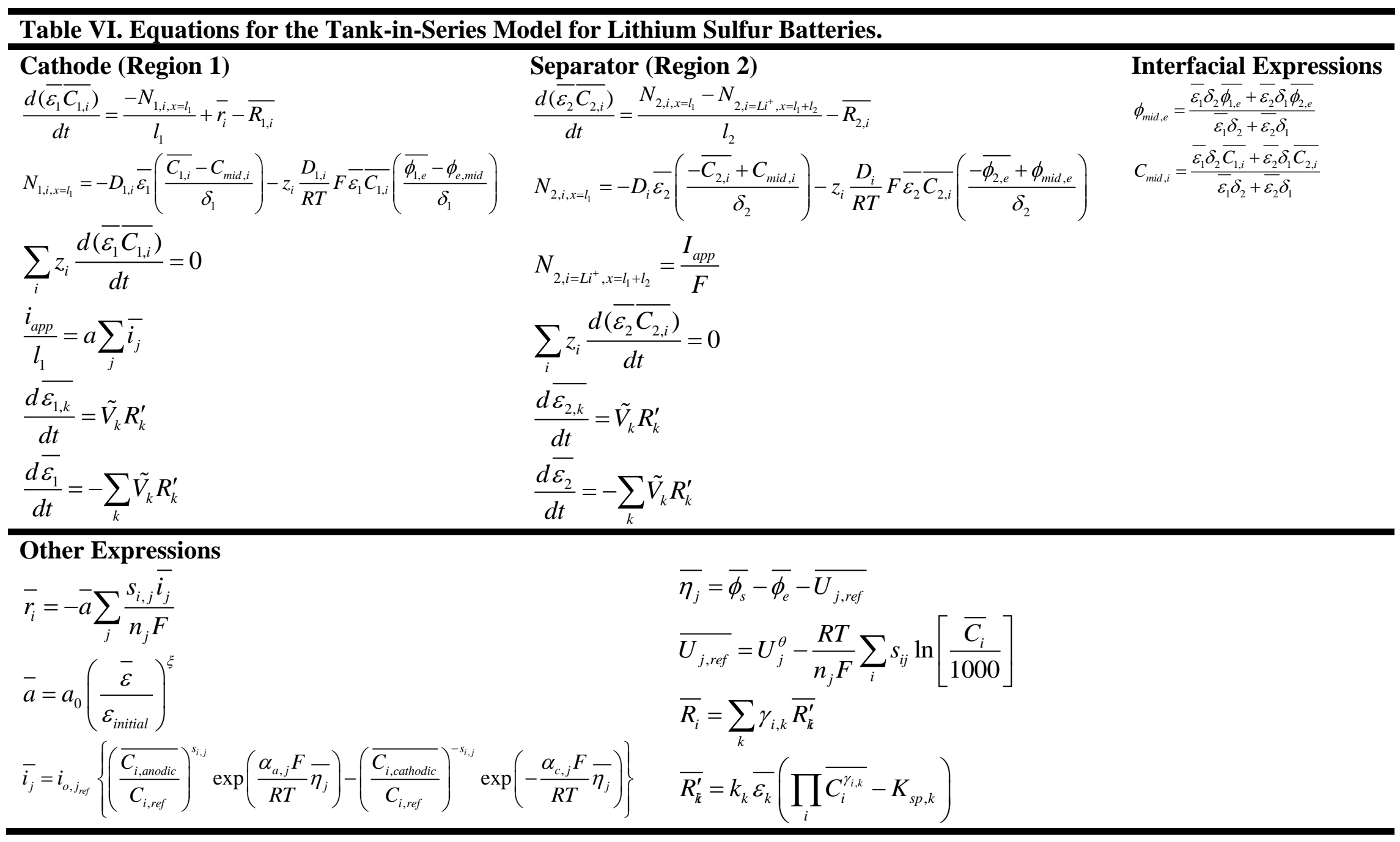




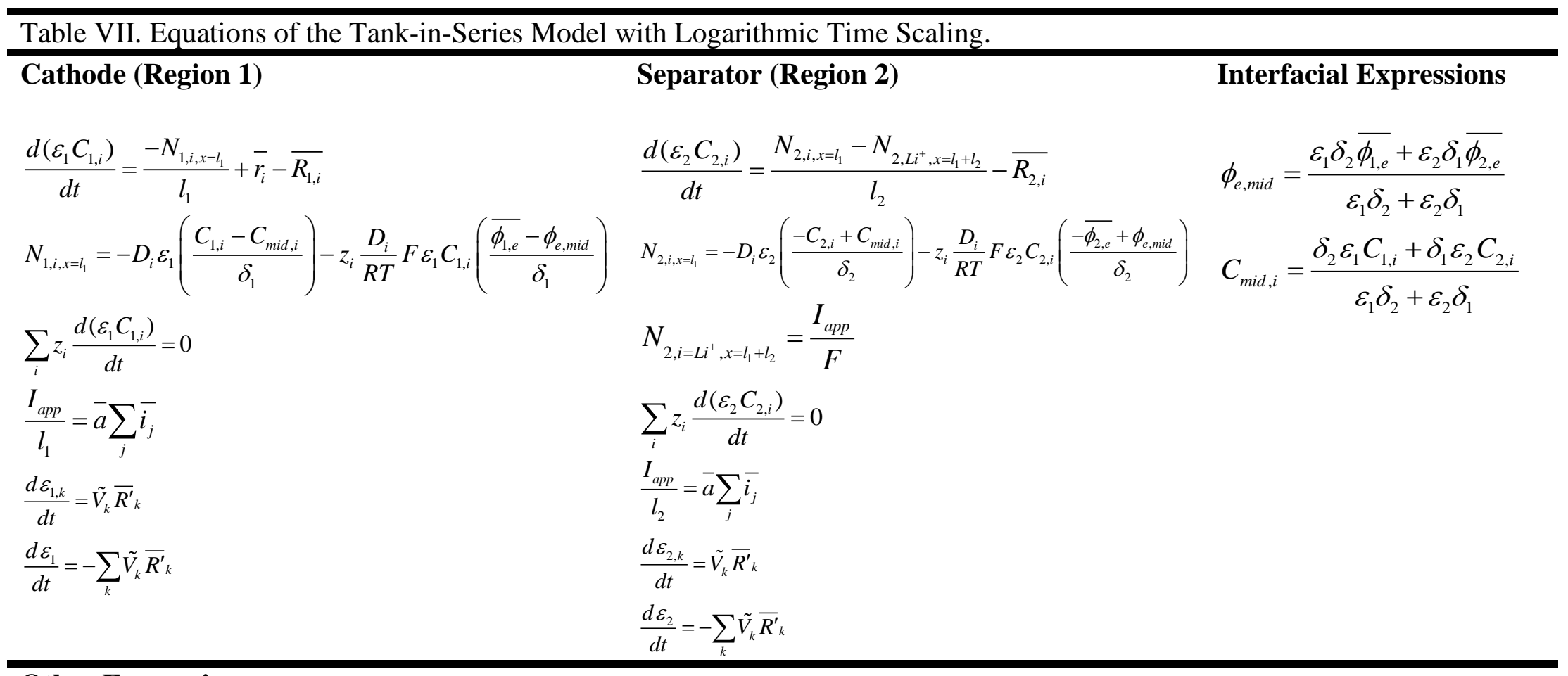

Other Expressions

$$
\begin{array}{ll}
\overline{r_{i}}=-\bar{a} \sum_{j} \frac{s_{i, j} \overline{i_{j}}}{n_{j} F} & \overline{\eta_{j}}=\overline{\phi_{s}}-\overline{\phi_{1, e}}-\overline{U_{j, r e f}} \\
\bar{a}=a_{0}\left(\frac{\varepsilon_{1}}{\varepsilon_{1, \text { initial }}}\right)^{\xi} & \overline{U_{j, r e f}}=U_{j}^{\theta}-\frac{R T}{n_{j} F} \sum_{i} s_{i j} \ln \left[\frac{C_{i}}{1000}\right] \\
\overline{i_{j}}=i_{o, j_{\text {ref }}}\left\{\left(\frac{C_{i, \text { anodic }}}{C_{i, \text { ref }}}\right)^{s_{i, j}} \exp \left(\frac{\alpha_{a, j} F}{R T} \overline{\eta_{j}}\right)-\left(\frac{C_{i, \text { cathodic }}}{C_{i, \text { ref }}}\right)^{-s_{i, j}} \exp \left(-\frac{\alpha_{c, j} F}{R T} \overline{\eta_{j}}\right)\right\} & \overline{R_{i}}=\sum_{k} \gamma_{i, k} \overline{R_{k}^{\prime}} \\
\hline
\end{array}
$$




\begin{tabular}{cc}
\hline $\begin{array}{c}\text { Table VIII. Values of the Parameter Study. } \\
\text { Parameter }\end{array}$ & Value \\
\hline C Rate & $0.2,0.5,1.0$ \\
Diffusion Coefficient $\left(\mathrm{m}^{2} / \mathrm{s}\right)$ & $1 \times 10^{-10}, 1 \times 10^{-11}, 1 \times$ \\
Cathode thickness $(\mu \mathrm{m})$ & $10^{-12}$ \\
Exchange current density, $i_{0 j}, j=2 . .6 *$ & 40,80 \\
\hline * not shown & $1 \times 10^{-4}, 1 \times 10^{-2}, 1 \times 10^{2}$ \\
\hline
\end{tabular}

